\title{
L'objet dans l'espace agricole. Le regard d'un géoagronome
}

\author{
Jean-Pierre Deffontaines \\ Géoagronome, directeur de recherche émérite INRA, 27 rue Anatole France, 92370 Chaville, France
}

«... l'objet est la signature humaine du monde.» Roland Barthes ${ }^{1}$.

À l'origine d'une approche de l'agriculture par les objets qu'elle crée, transforme, supprime ou conserve, et qu'elle présente à la vue, se situe la question des relations de l'agriculture au paysage.

La question revêt une importance croissante pour les politiques publiques, comme en atteste notamment l'introduction explicite du paysage dans la Loi d'orientation de 1999, mais aussi pour les diverses initiatives d'aménagement et de développement durable engagées dans le cadre de collectivités locales. Le paysage, par sa dualité de composante sensible du territoire et de support matériel de l'activité agricole, paraît pouvoir jouer un rôle de médiateur entre des usagers de plus en plus divers de l'espace rural et les praticiens, agriculteurs et éleveurs, qui participent à l'évolution du paysage. L'expérience montre que cette médiation par le paysage se fait difficilement, sans doute du fait que le paysage n'est pas une catégorie inscrite dans la culture des agriculteurs. La notion leur est souvent peu familière, ils l'estiment conçue par et pour les urbains.

Dans ce contexte, notre interrogation porte sur les possibilités de développer des liens et de fonder un dialogue entre usagers et producteurs du paysage ${ }^{2}$.

\footnotetext{
Auteur correspondant : jp.deffontaines@wanadoo.fr

1 Barthes, Roland, 1953. Les Planches de l'Encyclopédie, in Le Degré zéro de l'écriture. Nouveaux essais critiques, Paris, Le Seuil, 89-105.

2 Les usagers sont tous ceux, habitants et gens de passage, individus ou institutions, qui sont dans une relation souhaitée, indifférente ou subie avec le paysage. Les producteurs sont ici les agriculteurs et les éleveurs qui, parmi d'autres, participent à la dynamique paysagère. En fait, cette distinction traduit imparfaitement la réalité car les agriculteurs sont aussi des usagers, et les habitants et passants peuvent jouer un rôle sur le paysage. Nous retenons cependant la dualité simplificatrice des deux termes.
}

Elle renvoie à une question : y a-t-il des catégories auxquelles les agriculteurs se réfèrent qui les rapprochent du paysage?

Un premier détour vers le paysage se fait par le produit. Dans l'image du produit de qualité, pour fonder son identité territoriale, intervient le paysage. Le développement de l'expérience des AOC et la place croissante du paysage dans les critères des cahiers des charges traduisent son importance dans les débats du monde de la production. Nous pensons qu'un autre accès au paysage passe par une reconnaissance des objets visibles dans l'espace agricole et des enjeux qu'ils représentent en terme paysager. L'hypothèse s'appuie sur l'attention particulière que portent les agriculteurs à l'ensemble des objets qui composent la portion agricole du paysage et qui résultent des aménagements et des pratiques productives successives. Par ailleurs, l'usager du paysage, l'urbain dans son désir de campagne notamment, a une représentation globale du paysage où la production agricole occupe peu de place. Dans ce contexte, l'objet de l'espace agricole ne peut-il être médiateur, l'agriculteur faisant une démarche de l'objet vers le paysage et l'usager, du paysage vers l'objet? Telle est la question qui fonde cette réflexion sur les objets que l'agriculture inscrit dans le paysage.

La démarche proposée consiste à élaborer une base de connaissances sur ces objets en mobilisant des acquis de l'agronomie et de la géographie. Sur cette base peut ensuite être engagée l'analyse des relations des acteurs aux objets.

Le propos est structuré en trois volets. Le premier présente une réflexion sur la notion d'objet et propose une catégorisation de l'objet dans l'espace agricole. Un second volet aborde les relations qui s'établissent entre les objets et les processus de production mis en œuvre par les praticiens agricoles et esquisse une théorisation de ces relations. Enfin, le troisième volet fait retour à l'interrogation initiale sur le rôle des objets dans le développement de 
liens entre producteurs et usagers du paysage. Dans cette perspective, des travaux de recherche de diverses disciplines et des expériences sont à envisager, la réflexion sur les objets ne représentant qu'une étape.

\section{Une problématique de l'objet}

Le sens donné au terme objet est le suivant : toute chose, y compris les êtres animés, qui affecte la vue; chose solide ayant unité et indépendance et répondant à une certaine destination ${ }^{3}$. Dans l'infinité des objets observables dans le territoire, l'attention est portée sur les objets de l'espace agricole, c'est-à-dire sur les objets qui ont un lien avec les diverses opérations constitutives de l'activité agricole de production. La référence aux travaux de R. Barthes et de G. Simondon permet de préciser le point de vue développé sur l'objet.

\section{Catégories de l'objet}

Dans son analyse des planches de L'Encyclopédie ${ }^{4}$, R. Barthes parle d'une philosophie de l'objet. Celle-ci réfléchit à l'être de l'objet et opère à la fois " un recensement et une définition ». L'objet encyclopédique est saisi par l'image à trois niveaux : " anthologique », l'objet est isolé de son contexte ; "anecdotique », l'objet est inscrit dans une scène vivante avec d'autres objets ; " génétique ", l'image livre le trajet qui va de la matière à l'objet fini. "L'objet est ainsi cerné sous toutes ses catégories : tantôt il est, tantôt il est fait, tantôt enfin il fait. » Barthes introduit les notions de "trajet de l'objet », de la matière transformée à travers une série d'épisodes et celle de «message de l'objet qui n'est délivré qu'en situation ».

On peut faire le rapprochement de l'objet visible dans le paysage avec l'objet encyclopédique. En effet, dans l'espace agricole, l'objet se manifeste au regard, il a un usage et il présente une dynamique. La première catégorie, l'objet « est », renvoie à une désignation, à une dénomination, à une qualification par sa matière, sa forme, sa taille. La seconde, l'objet « est fait », concerne son histoire, son origine, sa transformation, sa durée. La troisième catégorie, l'objet «fait », se réfère à sa fonction et au rôle qu'il joue dans les processus de production, dans les systèmes techniques (ensemble organisé de lois, de règles et de choix impliqués dans l'agencement fonctionnel de terrains, de cheptel, d'équipement et de pratique) ${ }^{5}$.

\footnotetext{
${ }^{3}$ Objet: «"ce qui est placé devant" [...]. Toute chose, y compris les êtres animés, qui affecte les sens [...]. Chose solide ayant unité et indépendance et répondant à une certaine destination. » Le Petit Robert.

4 Barthes, R., op. cit.

5 Osty, Pl., Lardon, S., Sainte Marie, Ch., 1998. Comment analyser les transformations de l'activité productrice des agriculteurs? Propositions à partir des systèmes techniques de production, Étud. Rech. Syst. Agraire et Dv., 31, 397-413.
}

\section{L'objet technique (l'objet qui « fait » de Roland Barthes)}

Dans son ouvrage Du mode d'existence des objets techniques $^{6}$, G. Simondon propose une définition de l'objet technique et développe une réflexion sur les relations de l'homme à ces objets. Schématiquement, pour lui, l'objet technique est la machine et l'outil. Il est le résultat d'une «invention perpétuée », d'une évolution, d'un perfectionnement. L'évolution résulte du jeu de causes économiques et sociales. Il existe des lignées évolutives de familles d'objets techniques. Chaque objet est le siège d'un "schème pur de fonctionnement ». Tout objet technique remplit des fonctions. La fonction essentielle est celle de «transformateur». La définition des objets techniques de Simondon s'applique à une partie des objets tels qu'ils ont été définis précédemment (tout objet concret visible dans l'espace agricole et en liens avec la production agricole). Elle s'applique en effet aux objets qui peuvent être regroupés sous la rubrique de «matériel agricole». Il s'agit d'un vaste champ d'objets observables, en activité ou entreposés, qui sont autant d'indicateurs des systèmes techniques de production actuels et passés. Ainsi, la machine et l'outil agricoles «transforment » la structure du sol, l'état d'un peuplement végétal cultivé, le modelé d'un terrain, l'agencement des parcelles. La problématique de G. Simondon incite à pousser plus loin la réflexion sur la notion d'objet technique en agriculture. En effet, dans le domaine de l'activité agricole, on est confronté à des objets que l'on peut qualifier d'hybrides en ce sens qu'ils répondent à la fois à des logiques de fonctionnement technique et à celles du fonctionnement du vivant. Ainsi, un troupeau dans une exploitation semble répondre à la définition de l'objet technique donnée par G. Simondon. Il est lié à l'homme, qui construit progressivement une conformation et des aptitudes des animaux pour répondre à des fonctions. Son évolution s'appuie sur un "schème de fonctionnement » qui est un plan de sélection et un schéma d'alimentation et de conduite. Mais à la différence de la machine, le troupeau mobilise des règles de fonctionnement physiologique propres au vivant.

\section{L'objet façonné (l'objet qui « est fait » de R. Barthes)}

La notion d'objet technique «transformateur » met en lumière celle d'objet façonné. Ces objets résultent des opérations techniques agricoles, ils apparaissent au cours des itinéraires techniques. Plus généralement, ils sont issus du fonctionnement des systèmes techniques. À un instant donné, il est possible de différencier des objets façonnés par la matière qui les constitue et par les traits morphologiques et topologiques qui les caractérisent.

\footnotetext{
${ }^{6}$ Simondon, G., 1969. Du mode d'existence des objets techniques, Paris, Aubier.
} 
Les objets façonnés sont des matières mises en forme. En agriculture, la matière est minérale et vivante. Le matériau terre, par exemple, présente des caractères physionomiques de texture et de structure ${ }^{7}$. Il s'organise verticalement en horizon dans le sol et en surface en unités de terrain. Dans le champ, le travail du sol transforme la physionomie du terrain. Dans l'interchamp, le terrain est aménagé en fossé, en talus ou en banquette. Les limites de champ sont marquées par des murs, des terrasses, des clôtures. La matière prend forme dans les constructions de hangars, de silos, d'abris. À une autre échelle, la combinaison des terrains, la mosaïque des champs, le réseau de chemins, l'agencement des bâtiments, désignent le terroir. Le sol, le champ et le terroir, trois niveaux où s'élaborent des états successifs de la matière minérale. À chaque niveau, apparaissent des objets émergents (de la motte au parcellaire).

La matière de l'objet est aussi vivante : de la plante au peuplement végétal cultivé, et de l'animal au troupeau au niveau du champ, de la haie aux réseaux et îlots boisés au niveau du terroir.

La gamme de dimensions des objets façonnés qui demeurent visibles à un observateur dans un paysage va schématiquement de la touffe d'herbe au terroir. La forme des objets est ponctuelle, linéaire, en surface ou en volume. Ils se combinent pour composer des structures en semi, en réseau, en maillage, en mosaïque. Des objets s'emboîtent tels la motte de terre dans la bande de labour, la bande dans le champ, le champ dans le parcellaire, le parcellaire dans le bassin versant.

Les géographes ruraux ont proposé la notion de structure agraire pour désigner l'organisation dans l'espace $\mathrm{d}^{\prime}$ objets spatiaux ${ }^{8}$. La notion de structure agraire contribue à une classification et à une cartographie des paysages ${ }^{9}$, mais elle est trop globale pour prendre en compte et désigner l'agencement des objets façonnés de l'activité agricole.

\section{L'objet-forme}

Les objets qui s'inscrivent dans le territoire au cours $\mathrm{du}$ fonctionnement des systèmes techniques ont des formes et laissent des traces plus ou moins visibles pour l'observateur et plus ou moins durables. Les objets ont des formes propres qui correspondent à leur contour. Il n'y a pas d'objets (tels qu'ils ont été définis) sans formes, de plus les objets ont entre eux des rapports de position qui définissent des formes, des figures.

\footnotetext{
7 Hénin, S. (Ed.), 1960. Le Profil cultural : principes de physique $d u$ sol, Paris, Société d'édition des ingénieurs agricoles.

${ }^{8}$ Lebeau, R. (Ed.), 1986. Les Grands Types de structures agraires dans le monde, Paris, Masson.

9 Brunet, P., 2000. L'inventaire des paysages de BasseNormandie, In Actes du colloque Actions paysagères et acteurs territoriaux, Université de Poitiers.
}

Faut-il distinguer l'objet de la forme; ne suffit-il pas de désigner l'objet par sa forme? La forme d'un objet à un moment donné peut évoluer, s'estomper avec l'usage de l'objet et avec l'intervention de phénomènes naturels tels que le climat, les phénomènes biogéochimiques qui caractérisent les objets de nature. Une haie, par exemple, est un objet qui présente des formes diverses selon la période de l'année et selon les pratiques d'exploitation et d'entretien. Bien que objet et forme soient liés, il y a un message fourni par l'objet et un message spécifique de la forme. Il convient de conserver les deux termes d'objet et de forme, d'où l'expression objet-forme.

L'objet se transforme, se déforme, avec le temps jusqu'à disparaître. La trace est ce qui subsiste d'un objet lorsqu'il a disparu. La trace a une forme, elle-même évolutive, qui s'inscrit dans le territoire pour une certaine durée. Ainsi, la bande de labour s'estompe au cours de $l^{\prime}$ hiver, sa trace reste visible par un bombement plus ou moins accusé. D'un objet on observe soit sa forme propre, soit la forme de sa trace.

\section{Les objets de l'espace agricole dans leurs relations avec les processus de production}

Il n'existe pas, en agronomie, de systématique des objets-formes observables, comme c'est le cas pour d'autres disciplines telles l'architecture, la géomorpho$\operatorname{logie}^{10}$, la botanique (taxonomie et systématique des plantes par leur forme). Une démarche d'inventaire et de nomenclature présente l'intérêt de distinguer, de désigner, de dénommer et de regrouper les objets selon divers points de vue. Mais, pour une intelligibilité des objets, la référence à l'activité agricole de production est essentielle. Elle révèle l'origine, les usages et les fonctions de l'objet. De ces usages et fonctions dépend largement leur dynamique.

\section{Genèse et dynamique des objets dans l'espace agricole}

En agriculture, les objets-formes s'inscrivent dans le territoire au fur et à mesure que se déroule l'activité de production agricole. C'est au niveau des systèmes techniques, qu'ils soient mis en œuvre dans le cadre du champ, du territoire de l'exploitation ou d'une portion plus étendue du territoire, que peuvent être analysés les processus d'émergence des objets.

Les objets techniques (les outils et les machines) dans l'espace agricole de production traduisent des stratégies

\footnotetext{
10 Ollier, C.D., 1983. Land form description without stages names, Australian geografical studies, 73-80.
} 
d'équipement des agriculteurs. Issus du secteur industriel et de la fabrication artisanale, ces d'objets sont plus ou moins récents et remplissent des fonctions diverses. Il y a là un vaste champ d'objets, en action, en attente ou abandonnés qui jouent un rôle notable dans le paysage.

Une famille d'objets façonnés résulte de la mise en place, dans le territoire, de dispositifs. Ceux-ci sont des aménagements finalisés par des fonctions particulières, comme celles de l'accès, de la délimitation, de la protection, de la mise en défens, de la maîtrise de l'eau, du stockage... Une autre famille d'objets façonnés, dont la durée très variable ne dépasse pas le temps de la campagne, résulte des pratiques de conduite des systèmes techniques. C'est-à-dire des façons concrètes dont les agriculteurs et les éleveurs procèdent quotidiennement dans la mise en œuvre des techniques de production.

Le champ, unité de gestion, le plus souvent observable, est un objet-clé de l'espace agricole. Sa configuration résulte des stratégies d'acquisitions foncières, des partitions, des regroupements, des restructurations. Des objets façonnés qui occupent sa surface sont des dispositifs ; ainsi les ados, les rigoles, les fossés, pour assurer la maîtrise de l'eau, les terrasses pour corriger la pente. D'autres, sur la bordure du champ, jouent des rôles divers de limites de propriété, d'abris, de ressources (haies, talus, murs, clôtures, barrières, chemins...). Différents objets sont associés au champ qui servent à stocker, alimenter, protéger (hangar, arbre isolé... ).

Le champ est aussi le lieu où se déroule un «itinéraire technique », succession ordonnée d'opérations techniques au cours d'une campagne. Chaque opération met en mouvement des objets techniques qui travaillent le sol, traitent, récoltent, stockent, transportent. Chaque opération, selon les pratiques, crée de nouveaux objets façonnés, en transforme certains, en fait disparaître d'autres ; ainsi le troupeau au pâturage et les irrégularités de la surface fourragère liées au comportement alimentaire des animaux. À chaque étape, le champ présente une combinaison particulière d'objets. La notion d'itinéraire physionomique $^{11}$ fait pendant à celle d'itinéraire technique pour désigner la succession d'états physionomiques du champ, de sa végétation et de ses contours. Certains changements d'objets sont brusques, d'autres plus lents, dépendent de l'évolution naturelle des sols et de la végétation. Ces changements sont autant d'indicateurs visuels pour le praticien.

Les champs s'assemblent dans l'espace agricole de façons diverses. Une attention particulière est portée à leur agencement dans des portions locales du territoire et dans celui de l'exploitation agricole. À ces niveaux, le fonctionnement des systèmes techniques commande l'émergence d'objets. Des champs s'avèrent être le siège

\footnotetext{
11 Deffontaines, J.-P., 1995. Dynamique physionomique d'un paysage rural. Essai de modélisation de la composante agricole, Cahiers agricultures, 4, 436-439.
}

d'un même système de culture ou d'élevage. Des objetsdispositifs apparaissent pour la protection, pour la collecte, pour le stockage des grains ou des déjections, pour la circulation des biens et des gens. Dans un territoire, les systèmes techniques sont influencés par des contraintes communes d'environnement (bassins versants, sites de protection), par des organisations économiques (bassins de collectes, aires d'une AOC, superficies sous influence d'une CUMA ou d'une agglomération), par des facteurs particuliers du milieu physique (sol, climat, relief). La mise en œuvre plus ou moins organisée de ces systèmes techniques marque le territoire par des entités spatiales aux configurations particulières (auréoles autour d'un village $^{12}$, organisation linéaire le long d'un axe de circulation), par des dispositifs propres (implantation de haies, de bandes herbeuses cynégétiques, de silos) et par des pratiques semblables (cultures spéciales, irrigation...). La notion d'unités agrophysionomiques (UAP) ${ }^{13}$ est proposée pour désigner des portions du territoire agricole de relative égale apparence.

\section{Le temps des objets dans l'espace agricole}

Les objets de l'espace agricole répondent à des temporalités propres à l'activité de production. Elles sont quotidiennes (pour le troupeau regroupé pour la traite), saisonnières (pour les travaux culturaux et leurs empreintes territoriales), pluriannuelles (pour l'assolement qui est l'inscription d'une rotation culturale dans l'espace agricole), décennales, voire plus (pour nombre de dispositifs, pour les bâtiments, pour le projet de sélection des animaux d'un troupeau).

En fonctionnement, les objets techniques introduisent dans le paysage un temps particulier qui est celui des pratiques et du travail. Aux pratiques de la «traite au parc », de l'épandage de fumier, de la moisson, du fanage, correspondent des objets techniques spécifiques qui, par leur présence, leur circuit, leur vitesse de déplacement, donnent à voir dans le paysage le rythme et la cadence de l'activité agricole de production. Évoluant dans une portion particulière du territoire, ils font le lien entre le temps du travail et l'espace agricole. Les objets techniques révèlent également le temps des innovations techniques. Les appareils neufs, expression des dernières innovations, le matériel vétuste ou les équipements abandonnés souvent visibles aux abords des bâtiments agricoles remplissant parfois des fonctions pour lesquelles ils n'avaient pas été conçus, renvoient à la génération des machines et des outils, parfois aux débuts de la mécanisation agricole.

\footnotetext{
12 Benoît, M., 1990. La gestion territoriale de l'activité agricole dans un village lorrain, Mappemonde, 4 .

13 Thinon, P., Deffontaines, J.-P., 2001. Des entités spatiales significatives pour l'activité agricole et pour les enjeux environnementaux et paysagers. Contribution à une agronomie du territoire, Le Courrier de l'environnement, 44, 13-28.
} 


\begin{abstract}
Encadré 1
L'objet initial est produit par le système technique en cours. Dans ce cas, on peut observer l'influence de l'usage sur la forme initiale et proposer une certaine évaluation de la durabilité du système et de l'objet (exemple en Albanie de la construction récente et peu soignée de terrasses dont la durée paraît mal assurée). La terrasse est le résultat d'un système technique antérieur distinct, elle est alors un objet hérité. Celui-ci peut être archaïque ou anachronique. La terrasse archaïque présente une certaine compatibilité avec les systèmes en cours. Les agriculteurs s'arrangent avec elle en la conservant telle quelle ou en la modifiant (exemple des anciennes terrasses de cultures des versants vosgiens aujourd'hui en herbe). La terrasse héritée peut s'avérer un objet anachronique. Il y a alors discordance et incompatibilité avec les systèmes techniques. L'objet est ignoré et disparaît après être passé par le stade plus ou moins long de trace (exemple des anciennes terrasses de cultures dans la zone de pâturage de demi-saison dans les Alpes). La terrasse peut également être supprimée, dans ce cas toute trace apparente de l'objet disparaît (exemple du remodelage des pentes viticoles du Kaysersthul).

Dans l'histoire agraire, l'objet-terrasse peut passer d'une catégorie dans une autre. Un objet anachronique peut être réhabilité par changement de système ou parce qu'une nouvelle fonction est attribuée à l'objet (exemple des terrasses de Majorque reconstruites à la suite de la création d'un parc naturel régional).
\end{abstract}

Liés à l'histoire économique et sociale, les systèmes techniques, dans un territoire, évoluent; cette évolution est déterminante sur l'histoire des objets façonnés. Dans une approche par les objets façonnés, l'agriculture peut être considérée comme "une activité fondée sur le vivant qui produit des objets changeants dans une trame $\mathrm{d}^{\prime}$ objets durables ${ }^{14}{ }$. Le temps des objets façonnés dans l'espace agricole est très variable. Il va de l'andin de foin qui limite l'effet de la rosée nocturne, aux configurations foncières qui demeurent visibles et inscrites pour des générations. L'objet peut être durable et sa forme changeante, c'est le cas d'un troupeau au pâturage (analogie aux nuages ou aux fluides) ou d'une haie selon les saisons. La trace de l'objet peut durer des siècles ${ }^{15}$. Ainsi certains objets-formes «structurent l'espace au-delà de la période qui les a initiés ${ }^{16} »$. La probabilité d'une longue durée augmente avec la dimension de l'objet; ainsi la motte de terre et le parcellaire.

La question de la durabilité des objets dans le territoire renvoie clairement à la dynamique des activités agricoles. Pour illustrer ce lien, deux objets façonnés sont analysés. Le premier est un objet-dispositif particulier dont la fonction première est de limiter l'effet de la pente, la terrasse. Le second objet concerne l'assemblage des unités agrophysionomiques d'un finage et ses transformations au cours des cinquante dernières années.

L'objet-terrasse est observé dans ses relations avec les systèmes techniques ${ }^{17}$ (Encadré 1 ).

Une grande diversité de liens s'établit entre objets et systèmes techniques. Selon les systèmes, les objets

\footnotetext{
${ }_{14}$ Deffontaines, J.-P., 1994. L'agriculteur-artisan producteur de formes, Natures Sciences Sociétés, 2, 4, 337-342.

${ }^{15}$ Guilaine, J., 2001. La très longue durée. Histoires et paysages agraires. Géoarchéologie, archéobotanique, archéozoologie, Études rurales, 153-154.

${ }_{16}$ Chouquer, G., 2000. L'Étude des paysages. Essai de leurs formes et de leur histoire, Paris, Errances.

17 Deffontaines, J.-P., 2003. Le temps des objets dans le territoire agricole. Une approche géoagronomique. Communication au séminaire "Temps long, pratiques agricoles et territoire», INRA-SAD, Dijon.
}

se créent, se conservent, se transforment ou disparaissent. L'analyse révèle des stratégies différentes des praticiens face aux objets hérités. De ces stratégies dépendent en partie l'état du territoire, le devenir des paysages.

Les unités agrophysionomiques, portions de territoire de relative égale apparence, sont observées dans un finage lorrain de 1950 à $2000^{18}$ (Encadré 2) .

En cinquante ans, les objets façonnés que sont les UAP changent de forme et se réorganisent selon des structures différentes qui traduisent les profondes transformations de la société locale et des systèmes techniques. L'agencement local des UAP s'avère un indicateur visuel des dimensions collectives et territoriales de ces systèmes.

\section{Vers une théorie géoagronomique de l'objet dans l'espace agricole}

L'approche par les objets est proposée dans le cadre d'une problématique de la dynamique des relations entre le paysage et l'agriculture. La référence à l'analyse agronomique a pour but de formaliser les liens entre les connaissances acquises sur les mécanismes qui soustendent les façons de produire des agriculteurs et des éleveurs avec les objets associés à ces pratiques. Dans l'ensemble hétérogène d'objets qui composent l'espace agricole, on peut considérer les objets tels qu'ils sont. Cette approche par leur morphologie et par la matière qui les compose n'est pas dénuée d'intérêt, mais là n'est pas l'intérêt majeur d'une problématique de l'objet. Il se situe dans la connaissance de sa genèse et de ses fonctions. Une distinction significative est celle faite entre les objets techniques qui remplissent des fonctions de «transformateur », et ceux qui sont façonnés. Les objets techniques sont ceux qu'utilisent et mobilisent les agriculteurs et les éleveurs dans leur activité de production. Les objets façonnés sont ceux dont héritent les agriculteurs de l'histoire écologique, technique et sociale du territoire qu'ils exploitent, et ceux qu'ils créent et transforment par les

\footnotetext{
18 Benoit, M., 1990. La gestion territoriale de l'activité agricole dans un village lorrain, Mappemonde, 4, Gestion de l'espace rural. Des pratiques aux modèles.
} 


\section{Encadré 2}

En 1950, le modèle d'organisation est en étoile. Au centre, le village où se regroupent les sièges d'exploitations. La surface agricole est divisée en trois zones d'utilisation homogène, les soles. Dans chaque sole se localise l'ensemble des parcelles en fourrage annuel, l'année suivante en blé, la troisième année en céréales secondaires. La forêt est disposée aux limites du finage. Chaque sole représente une UAP.

En 1980, le modèle est concentrique. La réduction du nombre d'exploitations et le développement de l'élevage se sont traduits par la disparition des contraintes collectives et par une spécialisation des usages dans le territoire. Chaque agriculteur dispose à proximité du village de parcs pour les vaches laitières, plus loin ce sont les prés de fauche. À proximité de la forêt, les grands parcs à génisses alternent avec les cultures de maïs. Chaque auréole correspond à une UAP.

En 2000, il reste deux ou trois exploitations, les sièges se sont éloignés du village. L'augmentation des surfaces de chaque exploitation s'est traduite par des utilisations hors du finage. Le modèle d'organisation, dans le finage, demeure concentrique mais éclate en îlots à l'extérieur.

systèmes techniques qu'ils mettent en œuvre. Une différenciation essentielle entre les objets est faite selon les différents niveaux fonctionnels de l'activité auxquels ils sont associés. Cette différenciation permet de mettre en correspondance des concepts de la science agronomique avec des objets concrets issus de l'activité agricole de production. On peut esquisser une grille reliant ces concepts fonctionnels à des catégories visuelles qui combinent des objets dans le paysage. À l'itinéraire technique correspond l'itinéraire physionomique au niveau du champ ou d'un ensemble de champs. Les systèmes techniques, par leur combinaison ou leur similitude dans l'espace ou par la contiguïté de composantes spatiales déterminantes, délimitent dans un territoire des unités agrophysionomiques (UAP). Au niveau de l'exploitation agricole, le système technique n'est pas aisément observable dans le paysage. Une approche complémentaire par enquête s'impose pour une intelligibilité de la dynamique des objets.

Dans cette analyse des objets, les agronomes ont un rôle particulier à jouer en élaborant des modèles de la dynamique des objets issus des systèmes techniques et en fournissant une clé de compréhension des processus de production de ces objets. En s'appuyant sur cette base de connaissances, d'une part l'agriculteur peut faire comprendre sa participation à la dynamique de la composante agricole du paysage, d'autre part, l'usager peut saisir les enjeux que soulèvent ses propres revendications paysagères.

\section{L'objet médiateur entre agriculteurs et usagers du paysage?}

Un constat qu'il convient de confirmer est que les agriculteurs et les éleveurs ont une véritable culture des objets-formes qui les entourent. Ils utilisent de multiples termes pour désigner les objets qui résultent de la mise en œuvre de leurs pratiques. Nombreux parmi ces objets sont des indicateurs visuels qui participent à la définition des pratiques et à l'évaluation de leurs effets. Ils sont également très sensibles aux objets relativement durables hérités des systèmes techniques et des pratiques passées.
Selon que ces objets hérités sont compatibles avec les systèmes qu'ils mettent en œuvre ou qu'ils s'avèrent anachroniques, ils les conservent en l'état, les remodèlent, les abandonnent ou en créent de nouveaux. Mais l'attention portée par les agriculteurs aux objets visibles de l'espace agricole qui les environne ne se limite pas aux fonctions productives des objets. Ces objets remplissent des fonctions écologiques, historiques, sociales, ludiques, esthétiques, symboliques ${ }^{19}$. L'expression suivante d'un éleveur du Jura, dans la zone de l'AOC du Comté, est révélatrice de ces fonctions : «le territoire est notre langage » (il parle de territoire, non de paysage).

L'usager du paysage, quel que soit l'usage, est confronté aux objets visibles résultant de l'activité agricole et inscrite de façon plus ou moins durable dans le territoire. Devant un paysage agricole, l'usager est face à une véritable vitrine de la profession agricole. Il est rare, dans nos sociétés où le travail est le plus souvent occulté par les produits finis, qu'une profession ait une façade qui donne à voir son activité en train de se faire ou montre les objets et les traces associés. Le territoire agricole, visible par tous, a le caractère d'un espace public.

Mais quelle attention l'usager du paysage porte-t-il à ces objets? Par quels moyens les lui rendre intelligibles?

L'objet de l'espace agricole est commun à différents acteurs, il contribue à la dynamique matérielle du paysage et il participe à la relation sensible des acteurs au paysage.

Aider les agriculteurs à reconnaître le rôle de l'objet technique et de l'objet façonné dans la dynamique des paysages, proposer aux usagers une intelligibilité de l'objet de l'espace agricole : deux voies convergentes pour la concertation paysagère.

\footnotetext{
19 Une voie d'approche de ces autres fonctions est de considérer un objet particulier visible dans l'espace agricole et de recueillir auprès des praticiens les raisons de sa présence. C'est le cas de la haie dans la recherche intitulée Bocagement, reconstitution et protection du bocage (Lamarche, $\mathrm{H}$. (Ed.), 2003) issue du programme Politiques publiques et paysages du ministère de l'Écologie et du Développement durable.
} 